\title{
Branching dendrites with resonant membrane: A "sum-over-trips" approach
}

\author{
S. Coombes, Y. Timofeeva, and C-M. Svensson. \\ Department of Mathematical Sciences, University of Nottingham, Nottingham, NG7 2RD, UK. \\ G. J. Lord. \\ Department of Mathematics Heriot-Watt University, Edinburgh, EH14 4AS, UK. \\ K. Josić. \\ Department of Mathematics, University of Houston, Houston, Texas 77204-3008, USA. \\ S. J. Cox. \\ Computational and Applied Mathematics, Rice University Houston, Texas 77005, USA. \\ C. M. Colbert. \\ Biology and Biochemistry, University of Houston, Houston, Texas 77204-5001, USA.
}

April 27, 2007

\section{Abstract}

Dendrites form the major components of neurons. They are complex branching structures that receive and process thousands of synaptic inputs from other neurons. It is well known that dendritic morphology plays an important role in the function of dendrites. Another important contribution to the response characteristics of a single neuron comes from the intrinsic resonant properties of dendritic membrane. In this paper we combine the effects of dendritic branching and resonant membrane dynamics by generalising the "sum-over-trips" approach [Abbott, L.F., Fahri, E., Gutmann, S.: The path integral for dendritic trees. Biological Cybernetics 66, 49-60 (1991)]. To illustrate how this formalism can shed light on the role of architecture and resonances in determining neuronal output we consider dual recording and reconstruction data from a rat CA1 hippocampal pyramidal cell. Specifically we explore the way in which an $I_{h}$ current contributes to a voltage overshoot at the soma.

\footnotetext{
*e-mail: stephen.coombes@nottingham.ac.uk
}

\section{Introduction}

The dendrites of neurons often exhibit elaborate branching structures, as so wonderfully described in the book Dendrites [30]. These branching projections act to transfer electrical activity between synapses and the soma. Indeed the electrical and branching properties of dendrites are known to play a critical role in integrating synaptic inputs and in determining whether action potentials are generated at either the soma or other hot spots within the dendritic tree [22, 25]. Much insight into the contribution of the electrical properties of dendrites to neuronal function has come from theoretical work first developed by Rall. In this regard Rall's work on dendritic modelling is one of the more obvious success stories in the field of mathematical neuroscience. For a historical perspective on Rall's contributions in this area we refer the reader to the book by Segev et al. [29], and for a review of many of the mathematical techniques still being used today we recommend Tuckwell's book [33]. Although much 
of the more recent work on dendritic modelling has tended to focus on the dynamics of nonlinear voltagegated ion conductances (reviewed in [28]) it is important to recognise that the passive properties of the dendritic tree provide the fundamental substrate for dendritic dynamics, as emphasised in the recent review article of London and Häusser [19]. In fact it would be fair to extend the statement that passive electrical properties form the backbone of neuronal computation to also include the resonant properties of dendritic membrane. Many neurons exhibit resonances whereby subthreshold oscillatory behaviour is amplified for inputs at preferential frequencies. One illustrative example is that of hair cells from the sacculi of frogs, which are seismic receptors sensitive in the frequency range of 10-150 Hz. For these cells the ionic mechanism underlying resonance is known to involve both a $\mathrm{Ca}^{2+}$ and a $\mathrm{Ca}^{2+}$-activated $\mathrm{K}^{+}$current [11]. From a mathematical perspective Mauro et al. [23] have shown that a linearisation of such channel kinetics, about rest, may adequately describe the observed resonant dynamics. In the terminology of electrical engineering the resulting linear system has a membrane impedance that displays resonant-like behaviour due to the additional presence of inductances. This extends the more usual ' $R C^{\prime}$ ' circuit description of passive membrane to the so-called quasi-active or 'LRC' case. Further work by Koch and Poggio [16] showed how the response function for an arbitrary branched dendritic tree with quasi-active membrane could be calculated in the Laplace (frequency) domain. This approach generalised the original graphical calculus of Butz and Cowan [3], valid for passive dendritic geometries. Later work by Abbott et al. [2] showed how to calculate response functions for passive branched dendritic trees directly in the timedomain. The machinery to do this borrows heavily from the path-integral formalism for describing Brownian motion, and was used to give simple diagrammatic rules for obtaining the Green's function for a passive tree [1]. For a discussion of the computational advantages of this approach see Cao and Abbott [4]. As noted in [2] the path integral approach relies on the superposition principle and so should also be applica- ble to quasi-active membranes, since this is described by linear equations. It is precisely this problem that we address and solve in this paper. We present our results in the "sum-over-trips" language of Abbott et al. [2] and show how their rules for constructing the Green's function for a branched tree must be modified to account for resonant membrane.

In section 2 we briefly review the formalism describing quasi-active membranes, along the lines described in [15]. Next in section 3 we develop a "sumover-trips" formalism that can cope with quasi-active dendritic trees of arbitrary geometry. Not only does this extend the original work of Abbott et al. [2], it further allows for the treatment of dendritic sub-units connected to a soma as well as allowing for differing cell membrane properties on each dendritic segment. Using a reconstructed cell and dual recording data we show, in section 4 , that this work is directly relevant to understanding the dynamics of real neurons. Specifically we treat resonances associated with $I_{h}$ and show how they contribute to a voltage overshoot at the soma. Finally in section 5 we discuss natural extensions of the work in this paper.

\section{Unbranched resonant dendrite}

Here we review the theory of quasi-active membrane and show how it may be interpreted in the language of 'LRC' circuits, i.e. circuits with a resistor, capacitor and inductance in parallel. To start with consider a generic ionic membrane current of the form

$$
I=I\left(V, w_{1}, \ldots w_{N}\right)
$$

where $V$ is a voltage and the $w_{k}$ are gating variables that satisfy

$$
\tau_{k}(V) \dot{w}_{k}=w_{k, \infty}(V)-w_{k}, \quad k=1, \ldots, N .
$$

It is traditional to write $\tau_{k}(V)=\left(\alpha_{k}(V)+\beta_{k}(V)\right)^{-1}$, where $w_{k, \infty}(V)=\alpha_{k}(V) \tau_{k}(V)$. Now consider variations around some fixed point

$\left(V, w_{1}, \ldots w_{N}\right)=\left(V_{\mathrm{ss}}, w_{1, \infty}\left(V_{\mathrm{ss}}\right), \ldots, w_{N, \infty}\left(V_{\mathrm{ss}}\right)\right)$, so that

$$
\delta I=\frac{\delta V}{R}+\left.\sum_{k=1}^{N} \frac{\partial I}{\partial w_{k}}\right|_{\mathrm{ss}} \delta w_{k}
$$


where we introduce the resistance $R$ defined by $R^{-1}=$ $\partial I /\left.\partial V\right|_{\text {ss }}$, and the subscript ss denotes that quantities are evaluated at steady state. Using (2) we can write the evolution of the perturbations in the gating variable as

$$
\left(\frac{\mathrm{d}}{\mathrm{d} t}+\alpha_{k}+\beta_{k}\right) \delta w_{k}=\left[\frac{\mathrm{d} \alpha_{k}}{\mathrm{~d} V}-w_{k, \infty} \frac{\mathrm{d}\left(\alpha_{k}+\beta_{k}\right)}{\mathrm{d} V}\right] \delta V .
$$

We may now write (3) in the form

$$
\delta I=\frac{\delta V}{R}+\sum_{k=1}^{N} \delta I_{k}
$$

where

$$
\left(r_{k}+L_{k} \frac{\mathrm{d}}{\mathrm{d} t}\right) \delta I_{k}=\delta V
$$

Here

$$
\begin{aligned}
r_{k}^{-1} & =\left.\tau_{k} \frac{\partial I}{\partial w_{k}}\left[\frac{\mathrm{d} \alpha_{k}}{\mathrm{~d} V}-w_{k, \infty} \frac{\mathrm{d}\left(\alpha_{k}+\beta_{k}\right)}{\mathrm{d} V}\right]\right|_{\mathrm{ss}}, \\
L_{k} & =\tau_{k} r_{k} .
\end{aligned}
$$

Hence, for a small perturbation around the steady state, the current $I$ responds as though the resistance $R$ is in parallel with $N$ impedance lines. Each of these is a resistance $r_{k}$ that is itself in series with an inductance $L_{k}$. Such inductive terms account for the oscillatory overshoot commonly seen in response to depolarising current steps or even after the firing of an action potential. Koch terms this form of equivalent linear membrane circuit quasi-active to distinguish it from a truly active (i.e. nonlinear) membrane [15].

Now consider a general current balance equation in the form

$$
C \frac{\mathrm{d} V}{\mathrm{~d} t}=-g_{L}\left(V-V_{\mathrm{L}}\right)-I+I_{\mathrm{inj}}
$$

The linearised equations will be

$$
\begin{aligned}
C \frac{\mathrm{d} V}{\mathrm{~d} t} & =-\frac{V}{\widetilde{R}}-\sum_{k=1}^{N} I_{k}+I_{\mathrm{inj}}, & \frac{1}{\widetilde{R}}=g_{L}+\frac{1}{R}, \\
L_{k} \frac{\mathrm{d} I_{k}}{\mathrm{~d} t} & =-r_{k} I_{k}+V . &
\end{aligned}
$$

The steady state voltage satisfies $I\left(V_{\mathrm{ss}}, w_{1, \infty}\left(V_{\mathrm{ss}}\right), \ldots, w_{N, \infty}\left(V_{\mathrm{ss}}\right)\right)+g_{L}\left(V_{\mathrm{ss}}-V_{L}\right)=I_{\mathrm{inj}}$. Introducing the Laplace transform (with spectral parameter $\omega$ )

$$
f(\omega)=\int_{0}^{\infty} \mathrm{d} t \mathrm{e}^{-\omega t} f(t)
$$

we find that $V(\omega)=K(\omega) I_{\text {inj }}(\omega)$, where

$$
K(\omega)=\frac{\sum_{k=1}^{N} r_{k}+\omega L_{k}}{\left(C \omega+\widetilde{R}^{-1}\right)\left(\sum_{k=1}^{N} r_{k}+\omega L_{k}\right)+1} .
$$

We identify $K(\omega)$ as the impedance of the linearised system, and note that it is a ratio of two polynomials, with the denominator of order $N+1$, and the numerator of order $N$ (where $N$ is the number of gating variables). For example, the linearisation of the HodgkinHuxley model generates a bandpass filter with optimal response around $67 \mathrm{~Hz}$ (see for example [15]). The range of validity of the reductive process is limited to a few millivolts around the resting potential. Later in section 4 we will use this approach to calculate the equivalent 'LRC' circuit model for a membrane with a so-called $I_{h}$ current.

We are now in a position to treat the dynamics of an infinite unbranched passive dendritic cable model supplemented by resonant currents of the type just described. In section 3 we will show how the response of a tree can be built up in terms of this solution and an appropriate set of coefficients determined both by the geometry of the tree and the resonances on each segment of the tree. We take as our starting point the standard cable equation coupled to a set of resonant currents. Writing $V=V(X, t), X \in \mathbb{R}, t \geq 0$, as the dendritic voltage the resonant cable equation is

$$
\begin{aligned}
\frac{\partial V}{\partial t} & =-\frac{V}{\tau}+D \frac{\partial^{2} V}{\partial X^{2}}-\frac{1}{C}\left[\sum_{k} I_{k}-I_{\text {inj }}\right], \\
L_{k} \frac{\mathrm{d} I_{k}}{\mathrm{~d} t} & =-r_{k} I_{k}+V .
\end{aligned}
$$

Here $D$ is the cable diffusion coefficient and $\tau$ the (passive) cell membrane time constant. After Laplace transforming (14) we obtain the ODE

$$
\begin{aligned}
& -V_{X X}+\gamma^{2}(\omega) V \\
& =\frac{I_{\text {inj }}-\sum_{k} \frac{L_{k} I_{k}(X, t=0)}{\left(r_{k}+\omega L_{k}\right)}+C V(X, t=0)}{C D}, \\
\gamma^{2}(\omega) & =\frac{1}{D}\left[\frac{1}{\tau}+\omega+\frac{1}{C} \sum_{k} \frac{1}{r_{k}+\omega L_{k}}\right],
\end{aligned}
$$

where $V=V(X, \omega)$ and $I_{\text {inj }}=I_{\text {inj }}(X, \omega)$. Introducing a re-scaled space $x=\gamma(\omega) X$ gives

$$
-V_{x x}+V=A
$$


where

$$
\begin{aligned}
& A(x, \omega)=\frac{1}{C D \gamma^{2}(\omega)}\left[I_{\text {inj }}(x / \gamma(\omega), \omega)\right. \\
& \left.-\sum_{k} \frac{L_{k} I_{k}(x / \gamma(\omega), t=0)}{r_{k}+\omega L_{k}}+C V(x / \gamma(\omega), t=0)\right] .
\end{aligned}
$$

The Green's function of the operator $\left(1-\mathrm{d}_{x x}\right)$ is simply $H_{\infty}(x)=\mathrm{e}^{-|x|} / 2$, and we may write the general solution to (17) in the form

$$
V(x, \omega)=\int_{0}^{\infty} \mathrm{d} y H_{\infty}(x-y) A(y, \omega) .
$$

In original co-ordinates (and considering vanishing initial data) we have that

$$
V(X, \omega)=\int_{0}^{\infty} \mathrm{d} Y G_{\infty}(X-Y, \omega) I(Y, \omega),
$$

where $I(X, \omega)=I_{\mathrm{inj}}(X, \omega) / C$ and

$$
G_{\infty}(X, \omega)=\frac{H_{\infty}(\gamma(\omega) X)}{D \gamma(\omega)}=\frac{\mathrm{e}^{-\gamma(\omega)|X|}}{2 D \gamma(\omega)} .
$$

Performing the inverse Laplace transform gives

$$
V(X, t)=\int_{0}^{t} \mathrm{~d} s \int_{0}^{\infty} \mathrm{d} Y G_{\infty}(X-Y, t-s) I(Y, s),
$$

where $G_{\infty}(X, t)$, is the inverse Laplace transform of $G_{\infty}(X, \omega)$. Note that in the limit $r_{k} \rightarrow \infty$ we recover the purely passive system $\left(\gamma^{2}(\omega)=(1 / \tau+\omega) / D\right)$ with Green's function

$$
G_{\infty}(X, t)=\frac{\mathrm{e}^{-t / \tau}}{\sqrt{4 \pi D t}} \mathrm{e}^{-X^{2} /(4 D t)} \Theta(t) .
$$

Here $\Theta(t)$ is the Heaviside step function.

Next we will see how to treat a branched network of connected dendritic segments, each possessing its own resonant dynamics.

\section{Branching}

A caricature of a branched dendritic tree with resonant membrane is depicted in Fig. 1. We define a node as point where branch segments touch (i.e. the vertices of the graph describing the tree). Nodes that do not have child nodes will be called terminal nodes. A finite segment of the tree, labelled by $i$, is described by the dynamics

$$
\begin{aligned}
\frac{\partial V_{i}}{\partial t} & =-\frac{V_{i}}{\tau_{i}}+D_{i} \frac{\partial^{2} V_{i}}{\partial X^{2}}-\frac{1}{C_{i}}\left[\sum_{k} I_{k, i}-I_{\mathrm{inj}, i}\right], \\
L_{k, i} \frac{\mathrm{d} I_{k, i}}{\mathrm{~d} t} & =-r_{k, i} I_{k, i}+V_{i}, \quad 0 \leq X \leq \mathcal{L}_{i} .
\end{aligned}
$$

We may then specify the dynamics on a tree by ensuring the appropriate boundary conditions at all nodes and terminals. These are i) continuity of potential, and ii) conservation of current. If we choose the coordinates on all of the radiating branches so that the node is at the point $X=0$ then continuity of potential requires that

$$
V_{i}(0, t)=V_{j}(0, t),
$$

for all values of $i$ and $j$ corresponding to segments radiating from the node. Conservation of current gives

$$
\left.\sum_{j} \frac{1}{r_{j}} \frac{\partial V_{j}}{\partial X}\right|_{X=0}=0
$$

Here $r_{j}$ is the axial resistance on segment $j$ (in $\Omega / \mathrm{cm}$ ), and the sum is over all $j$ values corresponding to segments radiating from the node in question. At an open terminal we impose $V_{i}\left(\mathcal{L}_{i}, t\right)=0$ and at a closed end $\partial V_{i}(X, t) /\left.\partial X\right|_{X=L_{i}}=0$. After Laplace transforming (24) and rescaling we have that

$$
-\left(V_{i}\right)_{x x}+V_{i}=A_{i}, \quad 0<x<\mathcal{L}_{i}(\omega),
$$

where $\mathcal{L}_{i}(\omega)=\gamma_{i}(\omega) \mathcal{L}_{i}$ and

$$
\gamma_{i}^{2}(\omega)=\frac{1}{D_{i}}\left[\frac{1}{\tau_{i}}+\omega+\frac{1}{C_{i}} \sum_{k} \frac{1}{r_{k, i}+\omega L_{k, i}}\right],
$$

with

$$
\begin{aligned}
& A_{i}(x, \omega)=\frac{1}{C_{i} D_{i} \gamma_{i}^{2}(\omega)}\left[I_{\mathrm{inj}, i}\left(x / \gamma_{i}(\omega), \omega\right)\right. \\
& \left.-\sum_{k} \frac{L_{k, i} I_{k, i}\left(x / \gamma_{i}(\omega), t=0\right)}{r_{k, i}+\omega L_{k, i}}+C_{i} V_{i}\left(x / \gamma_{i}(\omega), t=0\right)\right] .
\end{aligned}
$$

We may write the general solution to (27) in the form

$$
V_{i}(x, \omega)=\sum_{j} \int_{0}^{\mathcal{L}_{j}(\omega)} \mathrm{d} y H_{i j}(x, y, \omega) A_{j}(y, \omega),
$$

where $H_{i j}(x, y, \omega)$ satisfies

$$
\left[1-d_{x x}\right] H_{i j}(x, y, \omega)=\delta_{i j} \delta(x-y)
$$

In addition $H_{i j}(x, y, \omega)$ must satisfy boundary conditions similar to those given above for $V_{i}$, namely

$$
\begin{gathered}
H_{k j}(0, y, \omega)=H_{m j}(0, y, \omega) \\
\left.\sum_{i} z_{i}(\omega) \frac{\partial H_{i j}(x, y, \omega)}{\partial x}\right|_{x=0}=0, \quad z_{i}(\omega)=\frac{\gamma_{i}(\omega)}{r_{i}} .
\end{gathered}
$$


Also at an open terminal node we require

$$
H_{i j}\left(\mathcal{L}_{i}(\omega), y, \omega\right)=0
$$

and at a closed terminal node

$$
\left.\frac{\partial H_{i j}(x, y, \omega)}{\partial x}\right|_{x=\mathcal{L}_{i}(\omega)}=0 .
$$

As it stands our discussion, and indeed that in [2], does not include the coupling of a tree to a soma. For passive trees one way to treat this is to consider equivalent cylinders connected to an isopotential soma, as in the work of Evans et al. [8,9]. To include this important special node we also treat the soma as an equipotential surface with a resistance $R_{s}$, capacitance $C_{s}$ and further include an inductive pathway described by $r_{s}$ and $L_{s}$. The membrane voltage in the soma $V_{s}$ has to

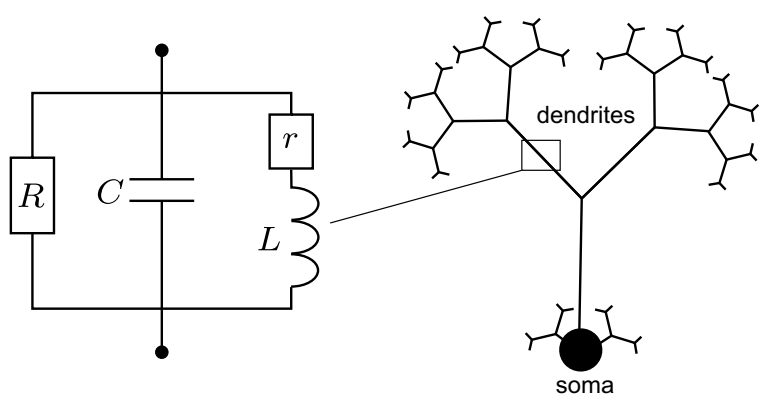

Figure 1: A caricature of a branched dendritic tree with resonant membrane. Each segment of the tree has its own resonant dynamics described by an 'LRC' circuit. The soma is regarded as a special node of the graph describing the dendritic tree.

satisfy the following equations

$$
\begin{aligned}
C_{s} \frac{\partial V_{s}}{\partial t} & =-\frac{V_{s}}{R_{s}}+\left.\sum_{j \in \Gamma} \frac{1}{r_{j}} \frac{\partial V_{j}}{\partial X_{j}}\right|_{X_{j}=0}-I_{s}, \\
L_{s} \frac{\mathrm{d} I_{s}}{\mathrm{~d} t} & =-r_{s} I_{s}+V_{s}
\end{aligned}
$$

with $V_{s}(t)=V_{j}(0, t)$ and $j \in \Gamma$, where $\Gamma$ is the set that indexes all of the branches attached to the soma. In this case $H_{i j}(x, y, \omega)$ must satisfy the further boundary condition

$$
\left.\sum_{k \in \Gamma} z_{k}(\omega) \frac{\partial H_{k j}(x, y, \omega)}{\partial x}\right|_{x=0}-\gamma_{s}(\omega) H_{k j}(0, y, \omega)=0,
$$

where

$$
\gamma_{s}(\omega)=C_{s} \omega+\frac{1}{R_{s}}+\frac{1}{r_{s}+\omega L_{s}} .
$$

We now seek a solution for $H_{i j}(x, y, \omega)$ in terms of the known response function $H_{\infty}$ and the labels $(i, j, x, y)$ and the frequency dependent parameters $\gamma_{k}(\omega)$, where $k$ indexes every segment in the tree. An infinite series expansion of this type that is consistent with (31) is

$$
H_{i j}(x, y, \omega)=\sum_{\text {trips }} A_{\text {trip }}(\omega) H_{\infty}\left(\mathcal{L}_{\text {trip }}\right) .
$$

Here $\mathcal{L}_{\text {trip }}=\mathcal{L}_{\text {trip }}(i, j, x, y, \omega)$ is the length of a path along the tree that starts at point $x=\gamma_{i}(\omega) X$ on branch $i$ and ends at the point $y=\gamma_{j}(\omega) Y$ on branch $j$. Note that on intermediate branches between branches $i$ and $j$, labelled by $k$ say, that distances are measured in terms of the scaled coordinates $\gamma_{k}(\omega) X, X \in\left[0, \mathcal{L}_{k}\right]$. We shall call these frequency dependent path lengths trips in analogy to the terminology used in the "sum-overtrips" formalism of Abbott et al. [2]. However, it is important to stress that in our case these trips depend upon the set of frequency dependent parameters $\gamma_{i}(\omega)$ that capture the resonant properties of the tree. As in [2] trips are constructed in accordance with the following rules:

1. A trip may start out from $\gamma_{i}(\omega) X$ by travelling in either direction along segment $i$, but it may subsequently change direction only at a node or a terminal. A trip may pass through the points $\gamma_{i}(\omega) X$ and $\gamma_{j}(\omega) Y$ but must begin at $\gamma_{i}(\omega) X$ on segment $i$ and end at $\gamma_{j}(\omega) Y$ on segment $j$.

2. When a trip arrives at a node, it may pass through the node to any other segment radiating from the node or it may reflect from the node back along the same segment on which it entered.

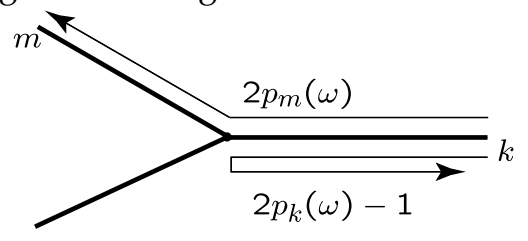

3. When it reaches a terminal, a trip always reflects back, reversing its direction.

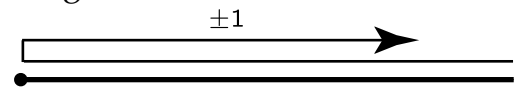


Every trip generates a term in (40) with $\mathcal{L}_{\text {trip }}$ given by summing the lengths of all the steps taken along the course of the trip. For example the four primary trips $\mathcal{L}_{\text {trip }}(i, j, x, y, \omega)$ on a simple dendritic tree consisting of two segments are $\gamma_{i}(\omega)\left(\mathcal{L}_{i}-X\right)+\gamma_{j}(\omega) Y$, $\gamma_{i}(\omega)\left(\mathcal{L}_{i}+X\right)+\gamma_{j}(\omega) Y, \gamma_{i}(\omega)\left(\mathcal{L}_{i}-X\right)+\gamma_{j}(\omega)\left(2 \mathcal{L}_{j}-Y\right)$ and $\gamma_{i}(\omega)\left(\mathcal{L}_{i}+X\right)+\gamma_{j}(\omega)\left(2 \mathcal{L}_{j}-Y\right)$ respectively. Note that all longer trips, even in a larger branched network, would consist only of constant additions to these four basic lengths. Hence, $\mathcal{L}_{\text {trip }}(i, j, x, y, \omega)$ is a linear function of $\pm x$, as required for $H_{\infty}\left(\mathcal{L}_{\text {trip }}\right)$ to be a solution to (31).

In appendix A we prove that (40) satisfies the required boundary conditions if the trip coefficients $A_{\text {trip }}(\omega)$ are chosen according to the following rules:

1. From any starting point $A_{\text {trip }}(\omega)=1$.

2. For every node at which the trip passes from an initial segment $k$ to a different segment $m(m \neq k)$ $A_{\text {trip }}(\omega)$ is multiplied by a factor $2 p_{m}(\omega)$.

3. For every node at which the trip enters along segment $k$ and then reflects off the node back along segment $k A_{\text {trip }}(\omega)$ is multiplied by a factor $2 p_{k}(\omega)-1$.

4. For every closed (open) terminal node $A_{\text {trip }}(\omega)$ is multiplied by a factor $+1(-1)$.

Here the frequency dependent parameters $p_{k}(\omega)$ are given as

$$
p_{k}(\omega)=\frac{z_{k}(\omega)}{\sum_{m} z_{m}(\omega)} .
$$

For a node describing the soma this coefficient takes the modified form

$$
p_{k}(\omega)=\frac{z_{k}(\omega)}{\sum_{m} z_{m}(\omega)+\gamma_{s}(\omega)} .
$$

Thus we arrive at the generalisation of the "sumover-trips" formalism that covers arbitrary resonant dendritic trees. In contrast to the original "sum-overtrips" formulation these arguments are developed in Laplace space and it remains to transform back to the temporal domain. To do this we first write $V_{i}(X, \omega)$ in the unscaled spatial coordinates as

$$
\sum_{j} \int_{0}^{\mathcal{L}_{j}} \mathrm{~d} Y H_{i j}\left(\gamma_{i}(\omega) X, \gamma_{j}(\omega) Y, \omega\right) \gamma_{j}(\omega) A_{j}\left(\gamma_{j}(\omega) Y, \omega\right) .
$$

Introducing

$G_{i j}(X, Y, \omega)=H_{i j}\left(\gamma_{i}(\omega) X, \gamma_{j}(\omega) Y, \omega\right) /\left(D_{j} \gamma_{j}(\omega)\right)$ we have that

$$
V_{i}(X, \omega)=\sum_{j} \int_{0}^{\mathcal{L}_{j}} \mathrm{~d} Y G_{i j}(X, Y, \omega)\left[U_{j}(Y, \omega)+I_{j}(Y, \omega)\right],
$$

where $I_{i}(X, \omega)=I_{\mathrm{inj}, i}(X, \omega) / C_{i}$ and

$$
U_{i}(X, \omega)=V_{i}(X, t=0)-\frac{1}{C_{i}} \sum_{k} \frac{L_{k, i} I_{k, i}(X, t=0)}{r_{k, i}+\omega L_{k, i}} .
$$

After taking the inverse Laplace transform of (44) we obtain

$$
\begin{gathered}
V_{i}(X, t)=\sum_{j}\left[\int_{0}^{\mathcal{L}_{j}} \mathrm{~d} Y G_{i j}(X, Y, t) U_{j}(Y, t=0)\right. \\
\left.+\int_{0}^{t} \mathrm{~d} s \int_{0}^{\mathcal{L}_{j}} \mathrm{~d} Y G_{i j}(X, Y, t-s) I_{j}(Y, s)\right] .
\end{gathered}
$$

Hence we identify the inverse Laplace transform of $G_{i j}(X, Y, w)$, namely $G_{i j}(X, Y, t)$, as the Green's function of the resonant dendritic tree. Note that we can build this function using a combination of the "sumover-trips" approach and a rescaling according to the resonant properties of each segment, before finally taking an inverse Laplace transform. Moreover, this naturally generalises the original approach of Abbott et al. [2] not only to resonant dendritic trees, but to those with different passive cell membrane properties on each segment (i.e. $\tau_{i}$ and $D_{i}$ differ across segments).

For the case that $\gamma_{k}(\omega)=\gamma_{m}(\omega)$ then $p_{k}(\omega)$ is independent of $\omega$. In this case for the choice $p_{k}=1 / 2$ a node acts no differently from a point on a single cable and we see that $r_{k}^{-1}=\sum_{m \neq k} r_{m}^{-1}$. Since the axial resistance $r_{k}$ scales as $d_{k}^{-2} \sqrt{D_{k}}$, where $d_{k}$ is the radius of the $k$ th branch, then we recover Rall's equivalent cylinder condition, $d_{k}^{3 / 2}=\sum_{m \neq k} d_{m}^{3 / 2}$, as first noted in [2]. Note that the rules for computing $G_{i j}(X, Y, \omega)$ require that trips start at $X$ and end at $Y$. To compute the function $G_{j i}(Y, X, \omega)$ the reverse trips from $Y$ to $X$ have to be used. If the trips are generated in reverse order the probability factors associated with crossing 
the nodes will be different. However, a simple calculation shows that the result of summing over reversed as opposed to original trips differs only by a constant factor $D_{i} r_{i} /\left(D_{j} r_{j}\right)$. This leads to the simple reciprocity identity

$$
G_{i j}(X, Y, t)=\frac{D_{i} r_{i}}{D_{j} r_{j}} G_{j i}(Y, X, t)
$$

In summary the Green's function for an arbitrary branched resonant dendritic tree is given by the Bromwich integral (inverse Laplace transform)

$$
\frac{1}{2 \pi i} \int_{c-i \infty}^{c+i \infty} \mathrm{d} \omega \mathrm{e}^{\omega t} \frac{H_{i j}\left(\gamma_{i}(\omega) X, \gamma_{j}(\omega) Y, \omega\right)}{D_{j} \gamma_{j}(\omega)} .
$$

Here, $\gamma_{i}(\omega)$ is given by (28) and $H_{i j}(X, Y)$ is given by (40). This last equation expresses $H_{i j}$ in terms of an infinite sum of terms involving the fundamental response function $H_{\infty}(x)=\mathrm{e}^{-|x|} / 2$ and the length of a frequency dependent 'trip'. The trip coefficients $A_{\text {trip }}(\omega)$ are generated according to the rules in $\mathrm{Ab}$ bott et al. [2], under the replacement of $p_{k}$ by $p_{k}(\omega)$ as given by (41) and (42). For any practical computational implementation the number of terms in the sum for $H_{i j}$ must be truncated. Since contributions of the individual terms for long trips (fixed $\omega$ ) are small, this truncation can be done by introducing a length cutoff. Numerical inversion of the Laplace transform can be efficiently performed using fast Fourier transforms. We have checked the validity of this approach by comparing code written in both $\mathrm{C}++$ and MATLAB with brute force simulations performed in NEURON [5] for a wide range of realistic neuronal geometries. Moreover, in many cases a good approximation is reached by including only the four shortest trips. In the next section we present some examples of this approach.

\section{Resonances associated with $I_{h}$}

Many neurons exhibit resonances whereby subthreshold oscillatory behaviour is amplified for inputs at preferential frequencies [13]. A nice example is that of the subthreshold frequency preference seen in neurons of rat sensorimotor cortex [12]. In response to suprathreshold inputs, this frequency preference leads to an increased likelihood of firing for stimulation near the resonant frequency. It is known that the nonlinear ionic current $I_{h}$ is partly responsible for this resonance. Indeed $I_{h}$ plays a variety of important roles in many neuronal and nonneuronal cell types [26], and it is believed that the presence of $I_{h}$ in dendrites could have a significant impact on the integration of subthreshold synaptic activity [21]. As such it is interesting to apply the framework we have developed above to the specific case of the $I_{h}$ current. Moreover, to gain insight into the resonant effects of $I_{h}$ it is useful to explore both idealised geometries and more realistic reconstructed cells.

Here we focus on the $I_{h}$ model of Magee [21], given by

$$
I_{h}=g_{h}\left(V-V_{h}\right) f,
$$

where $f$ is a single gating variable. The details of this model are given in Appendix B. In Figure 2 we plot the membrane voltage of an unbranched semi-infinite resonant dendrite, with a uniformly distributed nonlinear $I_{h}$ current, in response to a constant current injection of finite duration. Also shown is the plot of the voltage from the linearised model. The closeness of the two curves emphasises the usefulness of the quasiactive membrane description in approximating a fully nonlinear model of $I_{h}$.

\subsection{Idealised geometries I}

For the membrane with the 'LRC' circuit shown in Figure 1 (with a single inductance) the natural frequency is given by

$$
\omega^{*}=\frac{\sqrt{C L}-C r}{C L} .
$$

For an unbranched infinite resonant dendrite with a homogeneous distribution of electrical properties along its length the function $G_{\infty}(X, \omega)$ given by (21) will have its maximum at the frequency $\omega^{*}$ for any $X$. In contrast a branched system will not exhibit such a stimulus location independent property.

To study how branches with differing natural frequencies interact we first consider the case of two semi-infinite branches (with common passive parameters $\tau, D$ and $C$ ), with natural frequencies $\omega_{i}^{*}=$ 


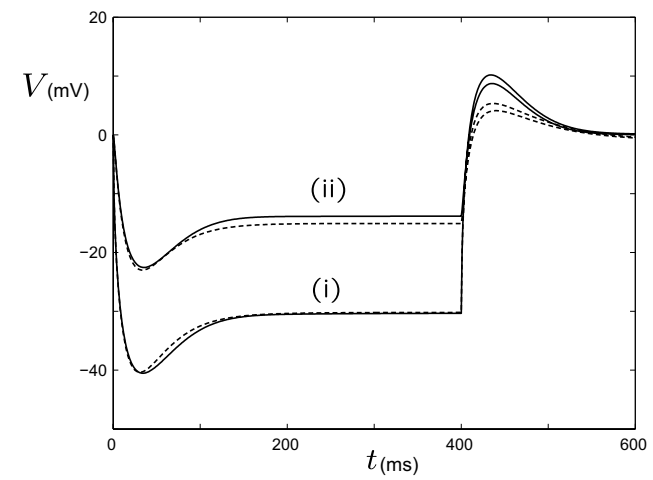

Figure 2: Membrane voltage of an unbranched semiinfinite resonant dendrite, at the location of the stimulus (i) and $500 \mu \mathrm{m}$ away from the point of stimulation (ii), in response to the current injection of amplitude $-0.3 \mathrm{nA}$ and duration $400 \mathrm{~ms}$. Passive parameters of the dendrite: $\tau=20 \mathrm{~ms}, D=50000 \mu \mathrm{m}^{2} / \mathrm{ms}$ and $C=1 \mu \mathrm{F} / \mathrm{cm}^{2}$. Dashed lines: the resonant membrane is modelled by the nonlinear $I_{h}$ current given by (49). Solid lines: the resonant membrane is modelled by the 'LRC' circuit with $r=13500 \Omega \cdot \mathrm{cm}^{2}$ and $L=1150 \mathrm{H} \cdot \mathrm{cm}^{2}$ (calculated using (7) and (8)).

$\left(\sqrt{C L_{i}}-C r_{i}\right) /\left(C L_{i}\right)$ connected together at $X=0$, such that $\omega_{2}^{*}>\omega_{1}^{*}$. The response functions $G_{11}(X, Y, \omega)$ and $G_{12}(X, Y, \omega)$ are easily constructed for an applied stimulus at location $Y$ on branch 1 and branch 2 respectively. The frequencies at which these functions reach their maximum define the resonant frequency of the (admittedly simple) dendritic tree, and thus satisfy $\partial G_{i j}(X, Y, \omega) / \partial \omega=0$. Note that the function $G_{12}(X, Y, \omega)$ consists of only one term $2 p_{2}(\omega) H_{\infty}\left(\gamma_{1}(\omega) X+\gamma_{2}(\omega) Y\right) /\left(D_{2} \gamma_{2}(\omega)\right)$, and the resonant frequency $\Omega$ of the tree satisfies

$$
\left(\gamma_{1}^{\prime} X+\gamma_{2}^{\prime} Y\right)\left(r_{1} \gamma_{2}+r_{2} \gamma_{1}\right)+r_{1} \gamma_{2}^{\prime}+r_{2} \gamma_{1}^{\prime}=0,
$$

where $\gamma_{i}=\gamma_{i}(\Omega)$ and $\gamma_{i}^{\prime}=\mathrm{d} \gamma_{i}(\Omega) / \mathrm{d} \omega$. The resonant frequency of the tree when the stimulus and response are taken on the same branch is obtained in a similar fashion (though the expression for $G_{11}(X, Y, \omega)$ consists of two terms). In Figure 3 we plot the resonant frequency of the tree, $\Omega$, with varying stimulus point $Y$ and fixed response point $X$. This figure nicely demonstrates that if the natural frequency of the branches differ, then the frequency with which the
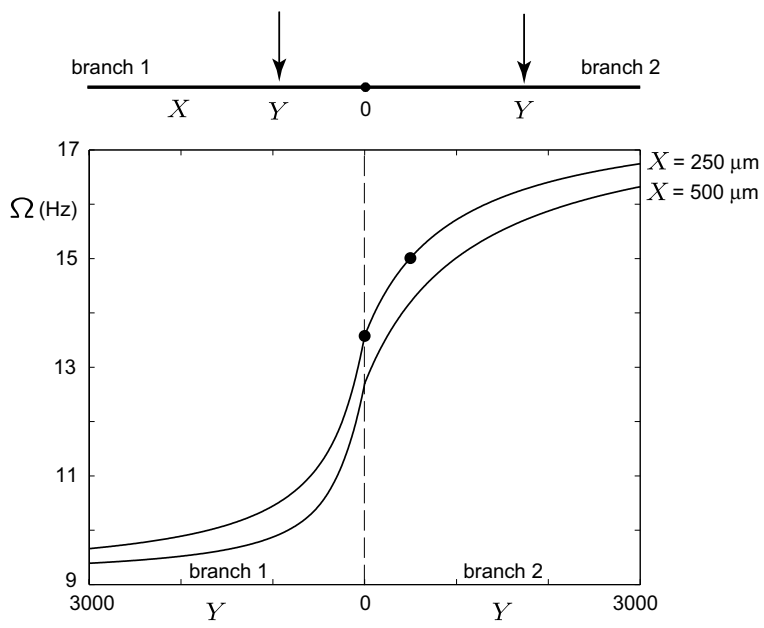

Figure 3: An idealised dendritic tree with two semiinfinite resonant branches. $\Omega$ is the resonant frequency of the whole tree as recorded at the locations $X=250 \mu \mathrm{m}$ and $X=500 \mu \mathrm{m}$ (on branch 1 ) as a function of the stimulus location $Y$ (which can be taken on either branch). Membrane parameters for each branch: $\tau_{1}=\tau_{2}=20 \mathrm{~ms}, D_{1}=D_{2}=50000 \mu \mathrm{m}^{2} / \mathrm{ms}$, $C_{1}=C_{2}=1 \mu \mathrm{F} / \mathrm{cm}^{2}$. The resistance and inductance of branch 1 are $r_{1}=27000 \Omega \cdot \mathrm{cm}^{2}$ and $L_{1}=2300 \mathrm{H} \cdot \mathrm{cm}^{2}$ respectively (with natural frequency $\omega_{1}^{*}=9.11 \mathrm{~Hz}$ ). The resistance and inductance of branch 2 are $r_{2}=13500$ $\Omega \cdot \mathrm{cm}^{2}$ and $L_{2}=1150 \mathrm{H} \cdot \mathrm{cm}^{2}$ respectively (with natural frequency $\omega_{2}^{*}=17.75 \mathrm{~Hz}$ ). Examples of voltage profiles in response to a current step for two cases marked by $(\bullet)$ are shown in Figure 4. 
whole tree responds depends on the location of the stimulus. Loosely speaking the response on branch 1 (far from branch 2) has a frequency more like that of $\omega_{1}^{*}$ when the stimulus is nearby, and a frequency more like that of $\omega_{2}^{*}$ when the stimulus is on the other branch and far away. Figure 4 shows examples of voltage responses and Green's function on branch 1 in response to the injected current on branch 2 .
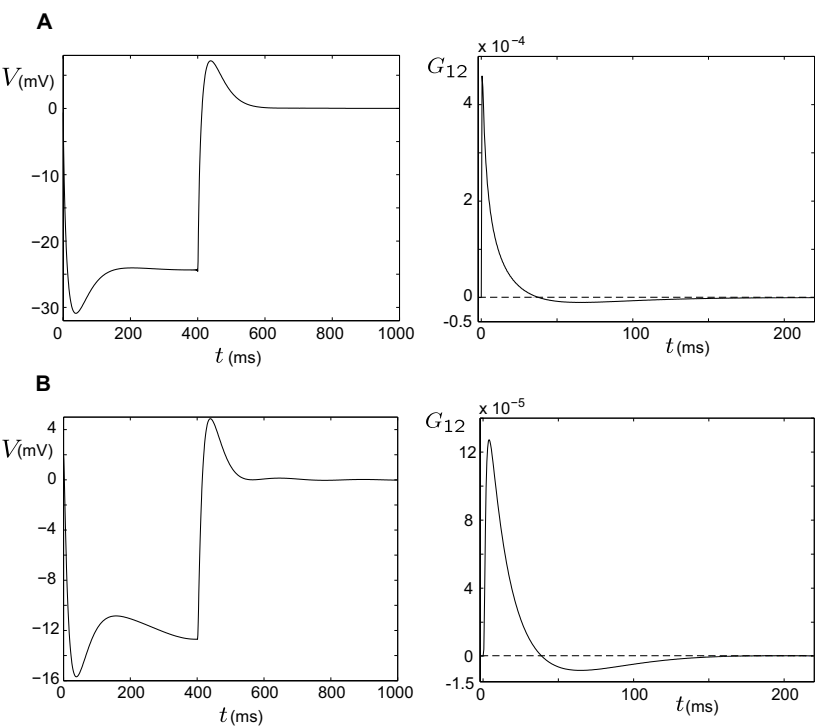

Figure 4: Examples of voltage profiles and Green's functions on branch 1 at the location $X=250 \mu \mathrm{m}$ in response to the current with amplitude $-0.3 \mathrm{nA}$ and duration of $400 \mathrm{~ms}$ injected on branch 2. A: Stimulus location is $Y=0 \mu \mathrm{m}$. B: Stimulus location is $Y=500$ $\mu \mathrm{m}$. Other parameters as in Figure 3.

\subsection{Idealised geometries II}

Here we consider the case of three semi-infinite dendrites emanating from a central node, as depicted in Figure 5. As before, the stimulus is applied to either branch 1 or branch 2 . The resonant frequency of the tree may be constructed in a similar fashion to that above. For example, the resonant frequency of the function $G_{12}(X, Y, \omega)$ is found by solving

$$
\begin{array}{r}
\left(\gamma_{1}^{\prime} X+\gamma_{2}^{\prime} Y\right)\left(r_{1} r_{3} \gamma_{2}+r_{2} r_{3} \gamma_{1}+r_{1} r_{2} \gamma_{3}\right) \\
+r_{1} r_{3} \gamma_{2}^{\prime}+r_{2} r_{3} \gamma_{1}^{\prime}+r_{1} r_{2} \gamma_{3}^{\prime}=0 .
\end{array}
$$

In Figure 5 we plot the resonant frequency of the tree as recorded on branch 1 in response to a stimulus (on branches 1 and 2) as the natural frequency on branch 3 is varied. Figure 5A shows an example where $\omega_{1}^{*}=\omega_{2}^{*}$. If $\omega_{3}^{*}$ is below some critical value, then we find that $\Omega=\omega_{1}^{*}=\omega_{2}^{*}$. Above this critical value then $\Omega$ increases toward $\omega_{3}^{*}$ (and more so when the stimulus point is close to branch 3). Figure 5B shows an example when the natural frequencies of branches 1 and 2 differ. If branch 3 is passive or only weakly resonant $\left(\omega_{3}^{*} \sim 0\right)$, then the system behaves much like that of the previous example (i.e. as a system of just two interacting resonant branches). As $\omega_{3}^{*}$ is increased from zero the resonant frequency, $\Omega$, of the tree (as measured on branch 1) increases toward $\omega_{3}^{*}$.

\subsection{Idealised geometries III}

Till now we have considered uniform distributions of conductances along a dendritic segment. However, it is now well known that dendritic trees can have nonuniform membrane conductances (see for example [20]). One way to treat spatial dependencies is to break a single segment into many pieces, each with a distinct yet uniform parameter set. A piecewise constant approximation to any spatially varying parameter can then be naturally implemented on this segmented cable. Using such an approach we now briefly turn to the observation that the conductance of $I_{h}$ increases with the distance from the soma in pyramidal neurons $[17,18]$.

A spatially varying conductance $g_{h}=g_{h}(X)$ leads to a spatially varying steady-state and from equations (7) and (8) to spatially varying forms for the resistance and inductance. Direct numerical simulations of a long cable with the full nonlinear $I_{h}$ model (49) show that the steady state voltage change is less than $1 \mathrm{mV}$ and the steady state change in the gating variable is less than 0.01 for a ten-fold magnification of $g_{h}$. Hence, it is reasonable to use the approximation $r^{-1}(X)=K g_{h}(X)$ and $L(X)=\tau r(X)$ (with the constant $K$ determined from (7) assuming a space-independent steady-state), for a piecewise constant function $g_{h}(X)$. Using a chain of 100 segments (for a dendrite of total 


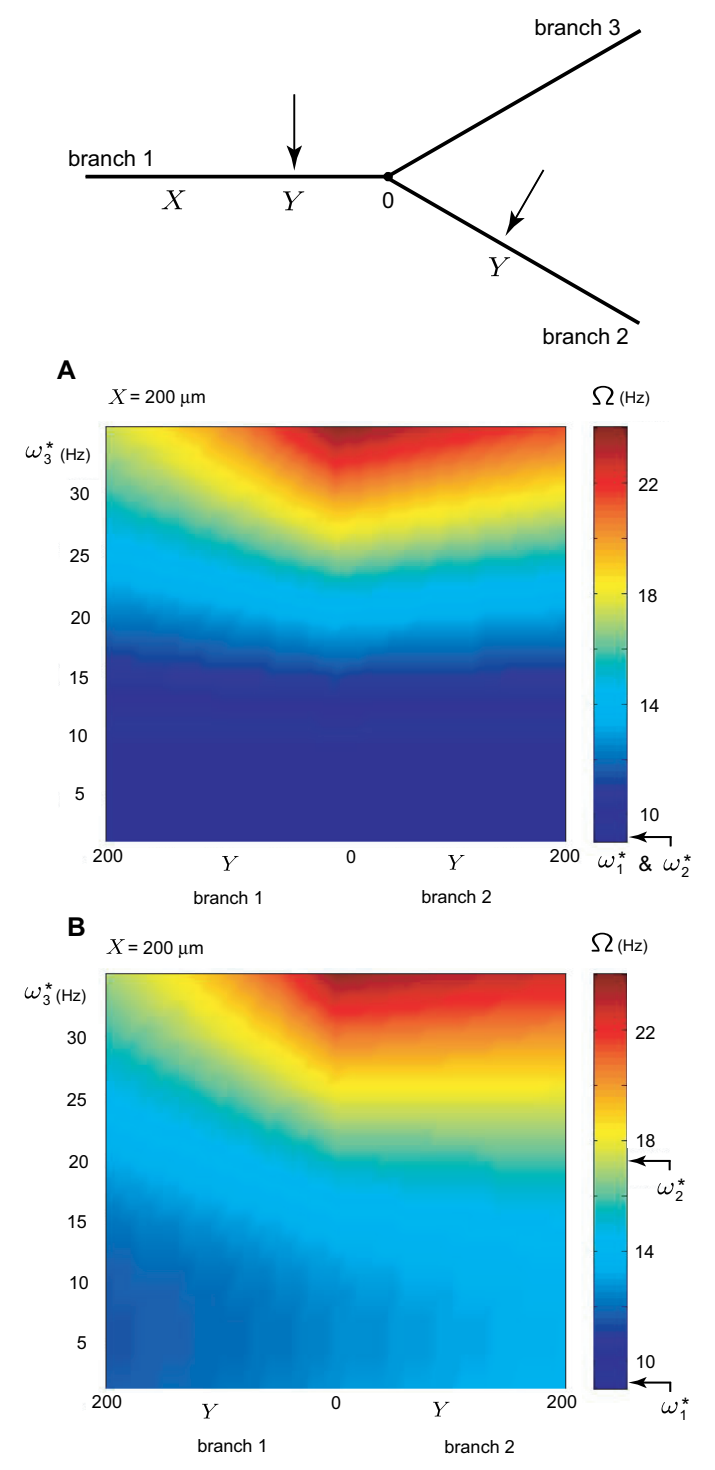

Figure 5: An idealised dendritic tree with three semiinfinite resonant branches. Here we plot the resonant frequency $\Omega$ of the dendritic tree as measured on branch 1 (at $X=200 \mu \mathrm{m}$ ), whilst varying both the natural frequency on branch 3 and the point of stimulus (on either branch 1 or branch 2). Passive membrane parameters for all three branches as in Figure 3. A: The resistances and inductances of branches 1 and 2 are the same and equal $r=27000 \Omega \cdot \mathrm{cm}^{2}$ and $L=2300$ $H \cdot \mathrm{cm}^{2}$ respectively. B: The resistance and inductance of branch 1 are $r_{1}=27000 \Omega \cdot \mathrm{cm}^{2}$ and $L_{1}=2300 \mathrm{H} \cdot \mathrm{cm}^{2}$ respectively. The resistance and inductance of branch 2 are $r_{2}=13500 \Omega \cdot \mathrm{cm}^{2}$ and $L_{2}=1150 \mathrm{H} \cdot \mathrm{cm}^{2}$ respectively. The resistance and inductance of branch 3 vary (between $67500 \Omega \cdot \mathrm{cm}^{2}$ and $5400 \Omega \cdot \mathrm{cm}^{2}$ for $r_{3}$ and between $5750 \mathrm{H} \cdot \mathrm{cm}^{2}$ and $460 \mathrm{H} \cdot \mathrm{cm}^{2}$ for $L_{3}$ ) for both A and B.

length $200 \mu \mathrm{m}$ ) we took a piecewise constant aproximation to the function $\bar{g}(1+3 X / 100)$ (suggested by data from [24]), for fixed $\bar{g}=0.05 \mathrm{mmho}^{-2}$, and considered the response at the soma $(X=0)$ to stimuli at increasing separation from the soma. In contrast to a spatially uniform conductance the time-to-peak of the signal at the soma remained approximately constant, whereas in the former case it increased with distance from the soma. In some sense we can view this as another example of how to achieve dendritic democracy [10], albeit this time for resonant dendrites.

In this example and the next the function $H_{i j}$ was constructed using four primary trips as well as their extensions generated by adding trips that start and end at the same point (as discussed in section 3 for the case of two connected segments). Longer trips generated from the primary trips were compared with the imposed length cutoff and ignored if $\mathcal{L}_{\text {trip }}(i, j, x, y, \omega)>6$ for fixed $\omega$. The convergence of the solution constructed according to the above procedure was validated by introducing increasing length cutoffs as well as by direct numerical simulations.

\subsection{A reconstructed cell}

Here we apply our formalism to a real neuronal geometry. We consider a rat CA1 hippocampal pyramidal cell, visualised with differential interference contrast optics using infrared illumination. The geometry of this cell was reconstructed (indicating the presence of 396 branches along with the soma) and saved in .hoc file format [5]. A diagram of the reconstructed cell is shown in Figure 6A. Dual simultaneous wholecell patch-clamp recordings were made in this cell and data were acquired at $5 \mathrm{KHz}$. Before sampling the data were filtered at $1 \mathrm{kHz}$ with an 8 pole filter. A pulse current of amplitude $-300 \mathrm{pA}$ and duration $400 \mathrm{~ms}$ (beginning at $10 \mathrm{~ms}$ ) was injected at the dendritic trunk. Dendritic and somatic recordings in response to this current injection are shown in Figure $6 \mathrm{~B}$, where the voltages are plotted with respect to rest (at about $-70 \mathrm{mV}$ ). The observed oscillatory voltage overshoot is believed to be associated with an $I_{h}$ current. In the corresponding quasi-active model with a 
single inductive current pathway (built using the reconstructed cell data) we take a uniform distribution of $I_{h}$ channel conductances. In this case the resistance $r$ and inductance $L$ of the resonant membrane are the same for all branches. Dendritic and somatic voltage responses in the model are shown by the solid lines in Figure 6B. The parameters $r$ and $L$ were fitted to the data, rather than assuming a specific model of $I_{h}$ and fixing them according to equations (7) and (8). As can be seen from the close agreement between theory and experiment in Figure 6 the "sum-over-trips" machinery generates quasi-active filters for realistic tree structures that can be used to organise and understand the structure of real dual electrode recording data.

\section{Discussion}

We have developed a "sum-over-trips" formalism for constructing the Green's function for a branched dendritic tree and soma with quasi-active membrane. This generalises the original work of Abbott et al. [2] for passive dendrites. Although in this paper we have focused on the ionic current $I_{h}$, we stress here that our approach can handle any channel kinetics written in the standard language of dynamic gating variables. Such a framework for studying (linear) dendritic neuron models obviates the need for the numerical solution of an underlying set of PDEs. One advantage of using the Green's function in comparison to a more standard compartmental approach is that this function has to be computed only once for a given dendritic structure. Thus changing the stimulation protocol does not require a whole new numerical simulation, rather just a convolution of the Green's function with the new input. In Laplace space at fixed frequency, the computational time will depend on the geometry of the tree, and issues of algorithmic efficiency reduce to those discussed in detail by Cao and Abbott for passive dendrites [4]. Although their approach can not treat resonant membrane our generalisation naturally does so with the introduction of a frequency parameter. The remaining computational overhead
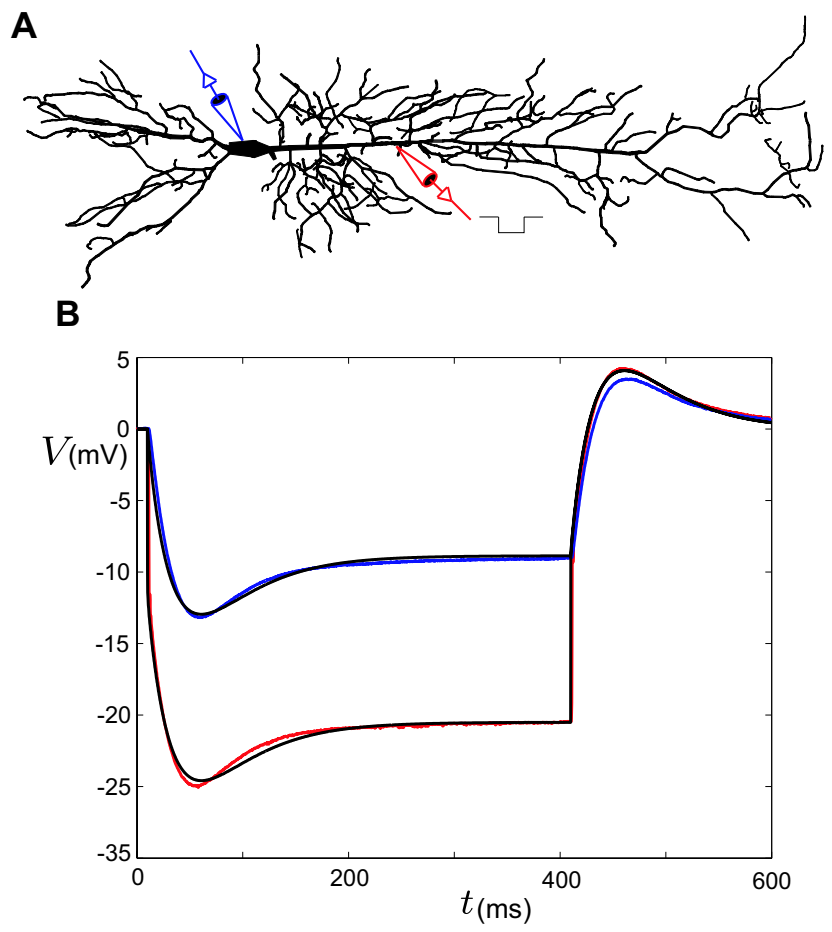

Figure 6: A: Reconstructed rat CA1 hippocampal pyramidal cell. B: An example of dendritic (red) and somatic (blue) dual simultaneous recording (with respect to rest) in response to the current injection at the dendrite trunk (average from 20 sweeps). A pulse current with amplitude $-300 \mathrm{pA}$ is applied for a duration of $400 \mathrm{~ms}$ starting from $10 \mathrm{~ms}$. The other two curves in B are dendritic and somatic voltage responses calculated from the model of the branched cell with resonant membrane. The model cell was stimulated at the dendrite (as shown in A) with the same current used in experimental recordings. Parameters across the tree: the specific cytoplasmic resistivity $R_{a}=100$ $\Omega \cdot \mathrm{cm}, C=1 \mu \mathrm{F} / \mathrm{cm}^{2}$ and the resistance across a unit area of pasive membrane $R_{m}=20000 \Omega \cdot \mathrm{cm}^{2}$. Thus $\tau=20 \mathrm{~ms}$ and diffusion coefficients $D_{i}$ vary from branch to branch. The conductance of $I_{h}$ is assumed to be uniform across the cell with the resistance and inductance of resonant membrane $r=24000 \Omega \cdot \mathrm{cm}^{2}$ and $L=2700 \mathrm{H} \cdot \mathrm{cm}^{2}$ respectively. Note that the voltage drop across the electrode's resistance is not fully compensated and the bridge balance is not corrected on the dendritic recording so that we shift our model results by an appropriate value of $10 \mathrm{mV}$. 
is then to perform a single inverse Laplace transform which can be done efficiently with standard numerical techniques. Moreover, our framework allows for a more analytical exploration of the way in which dendritic segments with differing natural frequencies contribute to an overall resonance at the level of the whole tree. It has also proved practical in use with real neuronal geometries. Indeed there is now a growing body of reconstructed cell data that can be uploaded from databases such as ModelDB ${ }^{1}$, and used within the framework we have developed here. Of course these geometries must be supplemented with data governing the distribution of active ionic conductances. Alternatively, with access to dual potential recording data, one may recover the quasi-active properties of dendritic neurons using the theory and algorithms developed by Cox and Griffith [6].

Two natural extensions of the work in this paper suggest themselves; i) to cover tapered dendrites, and ii) to cover active dendrites. If in the former case the underlying PDE model of the tapered model is linear (see [27] for a recent discussion of tapering) then there may be some hope to extend the "sum-overtrips" formalism. How to recover quasi-active properties from tapered dendrites is already known [7]. The problem of treating truly active dendrites [14] would seem at face value to be a substantially harder challenge. However, recent work on the spike-diffusespike model has shown how that a system of point hotspots embedded throughout a passive tree can provide a reasonable caricature of a tree with active conductances [31,32]. The solution of this model is expressed in part using the Green's function of the tree without hot-spots. Obviously this can be obtained with the "sum-over-trips" techniques we have described in this paper. Both of these extensions are topics of current research and will be reported upon elsewhere.

\section{Acknowledgements}

We would like to thank John Rinzel for pointing us to the work of Mauro et al. [23].

\footnotetext{
${ }^{1}$ http://senselab.med.yale.edu/senselab/modeldb
}

The work in this paper is supported through EPSRC Grant No. GR/S60914/01. SC would also like to acknowledge ongoing support from the EPSRC through the award of an Advanced Research Fellowship, Grant No. GR/R76219. CMC is supported by NIH grant R01 AG027577. Finally we would like to acknowledge support from the DTI in the form of a UK-Texas Biosciences Initiative award.

\section{Appendix A}

Using an extension of the argument in [1] we prove here that the the rules for generating the trip coefficients are consistent with the boundary conditions describing an arbitrary branched resonant dendritic tree.

Let $x$ denote the distance away from the node along the branch $k$ (see Fig. 7). The location of the stimulus $y$, the segment number $j$ and the variable $\omega$ are all considered to be arbitrary. Suppose that we sum all the trips starting from the node itself and ending at point $y$ on branch $j$. We denote the result of summing over all trips that initially leave the node along segment $k$ by $Q_{k j}(0, y, \omega)$. The result of all trips that leave the node along other branches $m$ is denoted by $Q_{m j}(0, y, \omega)$.

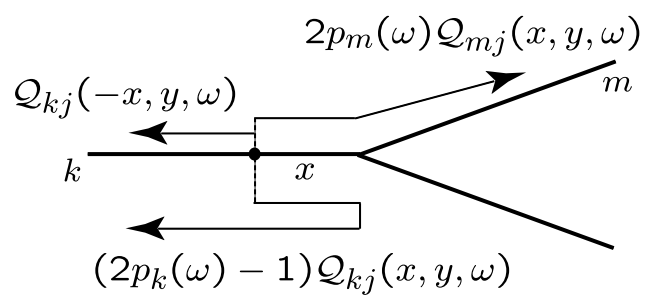

Figure 7: Main trips at a node.

Trips that start out from $x$ and move away from the node are identical to trips that start out from the node itself along segment $k$. The only difference is that the trips in the first case are shorter by the length $x$. We denote the sum of such shortened trips by $Q_{k j}(-x, y, \omega)$. The argument $-x$ means that a distance $x$ has to be subtracted from the length of each trip summed to compute $Q$ (not that the trips start at the point $-x)$. 
Trips that start out from $x$ by moving toward the node and then reflecting back along branch $k$ are also identical to trips that start out from the node along branch $k$ except that these are longer by the length $x$. In addition, because of the reflection from the node these trips pick up a factor $2 p_{k}(\omega)-1$ according to the "sum-over-trips" rules. Therefore, the contribution to the solution $H_{k j}(x, y, \omega)$ from those trips is $\left(2 p_{k}(\omega)-\right.$ 1) $Q_{k j}(x, y, \omega)$.

Finally, we have to take into account trips that start from $x$, move toward the node along branch $k$ and then leave the node by moving out along any of the radiating branches $m, m \neq k$. Crossing the node introduces a factor $2 p_{m}(\omega)$ and the sum of such trips is given by $2 p_{m}(\omega) Q_{m j}(x, y, \omega)$.

The full solution $H_{k j}(x, y, \omega)$ includes the contributions from all different types of trips we have been discussing. Thus,

$$
\begin{aligned}
H_{k j}(x, y, \omega) & =Q_{k j}(-x, y, \omega)+\left(2 p_{k}(\omega)-1\right) Q_{k j}(x, y, \omega) \\
& +\sum_{m \neq k} 2 p_{m}(\omega) Q_{m j}(x, y, \omega) .
\end{aligned}
$$

The functions $Q$ in this formula consist of infinite sums over trips, but we do not need to know what they are to show that the solution $H_{k j}(x, y, \omega)$ satisfies the node boundary conditions. At a node point we have

$$
H_{k j}(0, y, \omega)=\sum_{m} 2 p_{m}(\omega) Q_{m j}(0, y, \omega)
$$

The sum in the last formula is over all segments radiating from the node including branch $k$ and, thus, it shows that the solution at the point $x=0$ is independent of $k$. Therefore $H_{k j}(x, y, \omega)$ obeys the boundary condition (32).

To prove the boundary condition (33) we use equation (53) to find that

$$
\begin{aligned}
\left.\frac{\partial H_{k j}(x, y, \omega)}{\partial x}\right|_{x=0} & =\left.\sum_{m} 2 p_{m}(\omega) \frac{\partial Q_{m j}(x, y, \omega)}{\partial x}\right|_{x=0} \\
& -\left.2 \frac{\partial Q_{k j}(x, y, \omega)}{\partial x}\right|_{x=0} .
\end{aligned}
$$

Now we multiply this result by $p_{k}(\omega)$ and sum over $k$ to get

$$
\begin{aligned}
& \left.\sum_{k} p_{k}(\omega) \frac{\partial H_{k j}(x, y, \omega)}{\partial x}\right|_{x=0}= \\
& \left.\sum_{m} 2 p_{m}(\omega) \frac{\partial Q_{m j}(x, y, \omega)}{\partial x}\right|_{x=0}\left(\sum_{k} p_{k}(\omega)-1\right) .
\end{aligned}
$$

Using the property that $\sum_{k} p_{k}(\omega)=1$ we have

$$
\left.\sum_{k} p_{k}(\omega) \frac{\partial H_{k j}(x, y, \omega)}{\partial x}\right|_{x=0}=0 .
$$

Since $p_{k}(\omega)$ is proportional to $z_{k}(\omega)$ the solution $H_{k j}(x, y, \omega)$ satisfies the boundary condition (33).

A similar derivation can show that the "sum-overtrips" rules used at the terminals are also correct. If we consider a terminal point instead of a node point, $p_{m}(\omega)=0$ for $m \neq k$ in equation (53) and $p_{k}(\omega)=0$ for an open end or $p_{k}(\omega)=1$ for a closed end boundary condition at the terminal. Then equations (54) and (57) indicate that (34) and (35) are obeyed at all open and closed terminal nodes.

In the presence of the soma we have to check that the solution $H_{k j}(x, y, \omega)$ satisfies the corresponding boundary conditions (32) and (38). The proof that the solution satisfies the boundary condition (32) is identical to that for a node point. To show that the boundary condition (38) is also satisfied we use the following properties for $Q_{k j}(x, y, \omega)$, namely

$$
\begin{aligned}
& \frac{\partial Q_{k j}(x, y, \omega)}{\partial x}=-Q_{k j}(x, y, \omega), \\
& \frac{\partial Q_{k j}(-x, y, \omega)}{\partial x}=Q_{k j}(x, y, \omega) .
\end{aligned}
$$

These properties are a direct consequence of the form of $H_{\infty}(x)=\mathrm{e}^{-|x|} / 2$. We then have that

$$
\begin{aligned}
\left.\frac{\partial H_{k j}(x, y, \omega)}{\partial x}\right|_{x=0} & =-\sum_{m} 2 p_{m}(\omega) Q_{m j}(0, y, \omega) \\
& +2 Q_{k j}(0, y, \omega) .
\end{aligned}
$$

Note that, as compared to a simple node point, $p_{k}(\omega)$ for a branch radiating from the soma takes the modified form given by (42). By multiplying equation (60) by $z_{k}(\omega)$ and summing over $k$ we obtain

$$
\begin{aligned}
& \left.\sum_{k} z_{k}(\omega) \frac{\partial H_{k j}(x, y, \omega)}{\partial x}\right|_{x=0}= \\
& \sum_{m} 2 z_{m}(\omega) Q_{m j}(0, y, \omega)\left(1-\frac{\sum_{m} z_{m}(\omega)}{\sum_{m} z_{m}(\omega)+\gamma_{s}(\omega)}\right) .
\end{aligned}
$$


This gives us

$$
\begin{aligned}
& \left.\sum_{k} z_{k}(\omega) \frac{\partial H_{k j}(x, y, \omega)}{\partial x}\right|_{x=0}= \\
& \gamma_{s}(\omega) \sum_{m} \frac{2 z_{m}(\omega)}{\sum_{m} z_{m}(\omega)+\gamma_{s}(\omega)} Q_{m j}(0, y, \omega) .
\end{aligned}
$$

Using the equality (54) we recover the boundary condition (38).

\section{Appendix B}

Here we complete the details of the $I_{h}$ model given by (49) as described in [21]. The potential $V_{h}=-16 \mathrm{mV}$ and the conductance $g_{h}=0.09 \mathrm{mmho} \mathrm{cm}^{-2}$. The functions that appear in the gating dynamics are $f_{\infty}(V) \equiv$ $w_{\infty}(V), \alpha_{f}(V)=w_{\infty}(V) / \tau_{f}(V)$ and

$\beta_{f}(V)=\left(1-w_{\infty}(V)\right) / \tau_{f}(V)$. Here (for temperature $\left.27^{\circ} \mathrm{C}\right)$

$$
\begin{aligned}
\tau_{f}(V) & =\frac{\exp [0.03326(V+80)]}{0.00446(1+\exp [0.08316(V+80)])}, \\
w_{\infty}(V) & =\frac{1}{1+\exp [(V+92) / 8]} .
\end{aligned}
$$

\section{References}

[1] Abbott, L.F.: Simple diagrammatic rules for solving dendritic cable problems. Physica A 185(343356) (1992)

[2] Abbott, L.F., Fahri, E., Gutmann, S.: The path integral for dendritic trees. Biological Cybernetics 66, 49-60 (1991)

[3] Butz, E.G., Cowan, J.D.: Transient potentials in dendritic systems of arbitrary geometry. Biophysical Journal 14, 661-689 (1974)

[4] Cao, B.J., Abbott, L.F.: New computational method for cable theory problems. Biophysical Journal 64, 303-313 (1993)

[5] Carnevale, N.T., Hines, M.L.: The NEURON Book. Cambridge University Press (2006)

[6] Cox, S.J., Griffith, B.E.: Recovering quasi-active properties of dendritic neurons from dual poten- tial recordings. Journal of Computational Neuroscience 11, 95-110 (2001)

[7] Cox, S.J., Raol, J.H.: Recovering the passive properties of tapered dendrites from single and dual potential recordings. Mathematical Biosciences 190, 9-37 (2004)

[8] Evans, J.D., Kember, G.C., Major, G.: Techniques for obtaining analytical solutions to the multicylinder somatic shunt cable model for passive neurons. Biophysical Journal 63, 350-365 (1992)

[9] Evans, J.D., Kember, G.C., Major, G.: Techniques for the application of the analytical solutions to the multi-cylinder somatic shunt cable model for passive neurons. Mathematical Biosciences 125, 1-50 (1995)

[10] Häusser, M.: Dendritic democracy. Current Biology 11, R10-R12 (2001)

[11] Hudspeth, A.J., Lewis, R.S.: A model for electrical resonance and frequency tuning in saccular hair cells of the bull-frog, Rana Catesbeiana. Journal of Physiology 400, 275-297 (1988)

[12] Hutcheon, B., Miura, R.M., Puil, E.: Models of subthreshold membrane resonance in neocortical neurons. Journal of Neurophysiology 76, 698-714 (1996)

[13] Hutcheon, B., Yarom, Y.: Resonance, oscillation and the intrinsic frequency preferences of neurons. Trends in Neurosciences 23, 216-222 (2000)

[14] Johnson, D., Magee, J.C., Colbert, C.M., Christie, B.R.: Active properties of neuronl dendrites. Annual Reviews of Neuroscience 19, 165-186 (1996)

[15] Koch, C.: Cable theory in neurons with active, linearized membranes. Biological Cybernetics 50, 15-33 (1984)

[16] Koch, C., Poggio, T.: A simple algorithm for solving the cable equation in dendritic geometries of arbitrary geometry. Journal of Neuroscience Methods 12, 303-315 (1985) 
[17] Kole, M.H.P., Hallermann, S., Stuart, G.J.: Single $I_{h}$ channels in pyramidal neuron dendrites: Properties, distribution, and impact on action potential output. The Journal of Neuroscience 26(6), 1677-1687 (2006)

[18] Li, X., Ascoli, G.A.: Computational simulation of the input-output relationship in hippocampal pyramidal cells. Journal of Computational Neuroscience 21, 191-209 (2006)

[19] London, M., Häusser, M.: Dendritic computation. Annual Reviews of Neuroscience 28, 503$532(2005)$

[20] London, M., Meunier, C., Segev, I.: Signal transfer in passive dendrites with nonuniform membrane conductance. The Journal of Neuroscience 19, 8219-8233 (1999)

[21] Magee, J.C.: Dendritic hyperpolarizationactivated currents modify the integrative properties of hippocampal CA1 pyramidal neurons. Journal of Neuroscience 18, 7613-7624 (1998)

[22] Mainen, Z.F., Sejnowski, T.J.: Influence of dendritic structure on firing pattern in model neocortical neurons. Nature 382, 363-366 (1996)

[23] Mauro, A., Conti, F., Dodge, F., Schor, R.: Subthreshold behavior and phenomenological impedance of the squid giant axon. Journal of General Physiology 55, 497-523 (1970)

[24] Migliore, M., Ferrante, M., Ascoli, G.A.: Signal propagation in oblique dendrites of CA1 pyramidal cells. Journal of Neurophysiology 94, 41454155 (205)

[25] van Ooyen, A., Duijnhouwer, J., Remme, M.W.H., van Pelt, J.: The effect of dendritic topology on firing patterns in model neurons. Network 13, 311-325 (2002)

[26] Pape, H.C.: Queer current and pacemaker: the hyperpolarization activated cation current in neurons. Annual Review of Physiology 58, 299 327 (1996)
[27] Scott, A.: Neuroscience: A mathematical primer. Springer (2002)

[28] Segev, I., London, M.: Untangling dendrites with quantitative models. Science 290, 744-750 (2000)

[29] Segev, I., Rinzel, J., Shepherd, G.M. (eds.): The theoretical foundations of dendritic function: selected papers of Wilfrid Rall with commentaries. MIT Press (1995)

[30] Stuart, G., Spruston, N., Häusser, M. (eds.): Dendrites. Oxford University Press (1999)

[31] Timofeeva, Y., Lord, G.J., Coombes, S.: Dendritic cable with active spines: a modeling study in the spike-diffuse spike framework. Neurocomputing 69, 1058-1061 (2006)

[32] Timofeeva, Y., Lord, G.J., Coombes, S.: Spatiotemporal filtering properties of a dendritic cable with active spines. Journal of Computational Neuroscience 21, 293-306 (2006)

[33] Tuckwell, H.C.: Introduction to theoretical neurobiology volume I. Cambridge University Press (1988) 